\title{
自噬在缺血性脑卒中过程中的作用及其调节机制 研究进展
}

\author{
李玥 ${ }^{1}$, 郑婉晴 ${ }^{1}$, 潘玲 ${ }^{1}$, 刘孟茹 ${ }^{1}$, 周心雨 ${ }^{1}$, 陈忠 ${ }^{1,2}$, 张翔南 ${ }^{1 *}$ \\ 1. 浙江大学药理毒理研究所, 浙江大学药学院, 杭州 310058 ; \\ 2. 神经药理学与转化研究浙江省重点实验室, 浙江中医药大学药学院, 杭州 310053 \\ * 联系人, E-mail: xiangnan_zhang@zju.edu.cn \\ 收稿日期: 2021-07-14; 接受日期: 2021-08-06; 网络版发表日期: 2022-01-20
}

\begin{abstract}
摘要缺血性脑卒中是一种严重的脑血管疾病, 具有高发病率、高致残率和高死亡率的特点. 自噬是指细胞利用 溶酶体降解受损细胞器和大分子物质的过程. 缺血后脑内自噬被激活, 然而自噬在缺血性脑卒中过程中的作用尚 存争议. 近年来, 越来越多的证据提示自噬通过多种机制减缓了缺血性脑卒中损伤. 本文回顾了缺血性脑卒中诱 导自噬的主要病理因素, 探讨了自噬在缺血脑内的作用及其机制, 总结了缺血性脑卒中过程中自噬的调节剂, 以 期更新对缺血性脑损伤过程中自噬作用及其机制的认识, 为相关抗缺血性脑卒中药物的研究提供新的思路.
\end{abstract}

关键词 缺血性脑卒中, 自噬, 线粒体自噬

缺血性脑卒中通常由大脑中动脉栓塞、颅外颈内 动脉或椎动脉狭窄、闭塞或血栓形成、脑动脉痉挛、 心搏骤停等病因引起，具有高发病率、高致残率、高 复发率和高死亡率的特点，给患者家庭带来了严重的 经济和精神负担. 缺血性脑卒中病理机制复杂, 脑血管 梗死导致脑组织氧、糖供应不足，而后引发级联反应， 包括血脑屏障功能破坏、炎性细胞因子释放、氧化应 激水平增高、蛋白合成障碍以及线粒体代谢异常 等 ${ }^{[1 \sim 3]}$. 目前缺血性脑卒中的治疗主要包括溶栓以及神 经保护剂, 其临床效果有限, 新的缺血性脑卒中治疗策 略依赖于深入解析缺血性脑卒中关键病理机制. 自噬 是指细胞通过溶酶体清除受损细胞器以及大分子的过 程, 借此维持细胞本身的代谢稳态并完成细胞器的更
新 $^{[4]}$ (图1). 多项研究表明, 脑卒中后伴随着脑内细胞自 噬激活 ${ }^{[5]}$, 并受缺血时长、程度等因素影响. 自噬在卒 中脑内的作用尚存争议，一方面有证据表明自噬减少 了缺血性脑损伤 ${ }^{[9-14]}$; 另一方面, 自噬被认为是一种程 序性细胞死亡过程, 加重了神经损伤 ${ }^{[15-20]}$. 近年来, 越 来越多的研究提示自噬在缺血性脑卒中过程中, 特别 是在决定神经元存亡中发挥了重要作用 ${ }^{[21 ~ 23]}$, 然而自 噬参与脑缺血过程的细胞和分子机制仍有待深入研究.

本文总结了缺血性脑卒中的主要病理损伤机制, 综述了自噬在缺血性卒中脑内的作用, 进一步分析了 自噬的种类及其发生的分子机制，并阐述了缺血脑内 的选择性自噬及其作用以及缺血性脑卒中过程中自噬 的调节剂.

引用格式: 李玥, 郑婉晴, 潘玲, 等. 自潄在缺血性脑卒中过程中的作用及其调节机制研究进展. 中国科学: 生命科学, 2023, 53: 19-29

Li Y, Zheng W Q, Pan L, et al. The role of autophagy and related mechanisms in ischemic brain injury: an update (in Chinese). Sci Sin Vitae, 2023, 53: 19-29, doi: 10.1360/SSV-2021-0194 


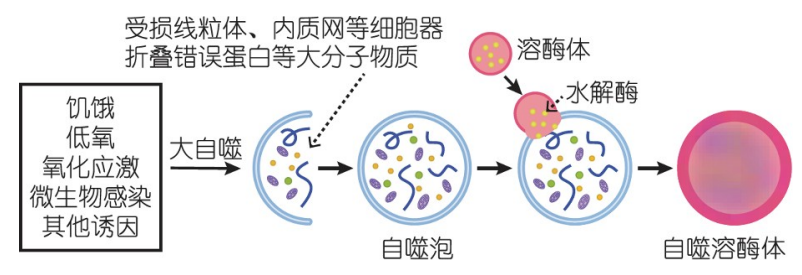

图 1 自噬的分子机制. 自噬是真核生物内一条重要的分解 代谢途径, 诸如饥饿、低氧、氧化应激、微生物感染以及其 他多种诱因均可诱导自噬发生. 自噬发生后, 受损线粒体、 内质网等细胞器及错误折叠蛋白等被自噬泡膜包裹, 自噬泡 膜进一步延伸形成较完整自噬泡后，将与溶酶体发生融合， 最后形成自噬溶酶体, 溶酶体中的溶解酶将自噬泡中的底物 降解(网络版彩图)

Figure 1 Molecular mechanism underlying autophagy. Autophagy plays an important role in catabolic pathways in eukaryotes. Starvation, hypoxia, oxidative stress, microbial infection, and other triggers induce autophagy. Subsequently, organelles, such as endoplasmic reticulum and mitochondria, and misfolded proteins can be enveloped by autophagic vesicles for degradation. The membranes of autophagosomes are further extended during maturation and fuse with lysosomes to form autolysosomes. Finally, lysosomes degrade the cargo in the autolysosomes (color online)

\section{1 脑缺血诱发自噬的病理因素}

缺血性脑卒中病理机制复杂，已有多篇综述对其 主要病理机制进行了总结 ${ }^{[24-26]}$. 在缺血脑内, 血流中 断造成脑组织氧气和葡萄糖供应中断，进而引发兴奋 性毒性、细胞调亡、神经炎症以及氧化应激等病理生 理事件. 这些过程严重影响了神经元、神经胶质细胞 及内皮细胞稳态，促进缺血性脑损伤。缺血后神经元 释放大量谷氨酸, 过度激活 $\mathrm{N}$-甲基 $\mathrm{D}$-天冬氨酸受体 (N-methyl-D-aspartate receptors, NMDARs)等谷氨酸 受体, 引发䥻离子内流, 造成神经细胞钻超载, 导致神 经元兴奋性毒性 ${ }^{[27]}$. 兴奋性损伤发生极快, 通常被认 为是急性脑缺血损伤的主要原因. 虽然减少兴奋性损 伤的神经保护剂可调节细胞自噬，但兴奋性损伤是否 直接影响自噬激活尚缺乏证据. 缺血后的亚急性期至 更长一段时间内，神经细胞可通过线粒体依赖及死亡 受体依赖的途径发生调亡 ${ }^{[28]}$. 有证据表明, 自噬通过 清除损伤线粒体，即线粒体自噬过程，减少神经细胞 调亡 ${ }^{[29]}$. 缺血及缺血后的血流复灌过程均会导致脑内 氧化与抗氧化平衡失调, 继而引发氧化应激, 其中 $\mathrm{NADPH}$ 氧化酶、黄嘌呤氧化酶及损伤线粒体都是活 性氧(reactive oxygen species, ROS)的重要来源. 脑缺 血再灌注后, 琥珀酸大量聚集, 并快速被琥珀酸脱氢酶 氧化, 继而大量电子通过线粒体复合物 I 逆向传递, 从
而导致线粒体来源ROS大量累积, 诱发氧化应激 ${ }^{[30]}$. 氧 化应激被认为是激活自噬的关键因素之一 ${ }^{[31]}$, 氧化应 激启动核因子NF-E2相关因子(nuclear factor-erythroid 2-related factor 2, Nrf2)诱导多个抗氧化酶表达 ${ }^{[32]}$, 而 $\mathrm{Nrf2}$ 被认为与自噬蛋白P62结合, 进而调控自噬 ${ }^{[33]}$. 此 外, 氧化应激也可能通过其他途径诱导自噬发生 ${ }^{[34]}$, 包括低氧诱导因子1(hypoxia-inducible factor 1, HIF1)Bcl-2/腺病毒E1B相互作用蛋白3(Bcl-2/adenovirus E1B interacting protein 3, BNIP3)和Bcl-2/腺病毒E1B $19 \mathrm{kD}$ 相互作用相似蛋白3(Bcl-2/adenovirus E1B-19 kD-interacting protein 3 like, BNIP3L)途径 ${ }^{[3537]}$; ROS-p53-糖酵 解和调亡调节因子 (glycolysis and apoptosis regulator, TIGAR)/损伤调控自噬调节因子(damage-regulated autophagy modulator, DRAM) 途径 ${ }^{[38-40]}$. 缺血引发神经炎 症，促进脑缺血损伤 ${ }^{[41]}$. 激活的胶质细胞分泌多种炎 症因子(如TNF- $\alpha, I L-1 \beta, I L-6$ ) 和促炎物质(如前列腺 素, ROS, NO). 特定的炎症因子可能通过其受体促进 神经细胞自噬发生 ${ }^{[42]}$. 另有报道发现, 在巨噬细胞过 表达NLRP3将诱导自噬激活，从而促进NLRP3 降解, 抑制下游炎症产物胱天蛋白酶 1 和IL- $1 \beta$ 的表达 ${ }^{[43]}$. 此 外, 有报道指出, 自噬通过清除炎症小体进而减轻缺 血后的神经炎症 ${ }^{[44]}$. 总之, 现有的证据表明, 脑缺血后 的多种病理生理因素均参与调控神经细胞的自噬, 并 可能影响缺血损伤过程.

\section{2 缺血脑内自噬发生的细胞类型及其作用}

缺血性脑卒中可激活多种脑内细胞自噬发生, 包 括神经元细胞、小胶质细胞、星形胶质细胞和内皮细 胞. 然而, 自噬在缺血性脑损伤中的作用仍存在争议.

\section{1 神经元中的自噬}

神经元是神经系统最基本的结构和功能单位，在 缺血性脑卒中过程中, 神经元的不可逆损伤直接导致 神经功能障碍. 早在 20 世纪90年代就有研究发现, 缺 血损伤后海马CA1(cornuammonis1)区神经元存在含有 复杂成分的空泡 ${ }^{[45]}$. 此后多个研究发现脑缺血后大脑 皮层神经元胞质内出现空泡结构. 利用GFP(green fluorescent protein)-LC3(microtubule associated protein 1 light chain 3)转基因小鼠的短暂性大脑中动脉栓塞 (transient middle cerebral artery occlusion, tMCAO)模 
型, 发现自噬泡(LC3 苂光)主要集中于神经元而非胶质 细胞中，提示脑缺血损伤后自噬可能主要发生在神经 元 $^{[46]}$. 然而, 自噬对缺血神经元的作用长期以来一直 存在争议. 部分研究认为, 自噬促进了脑缺血引起的 神经元死亡: 在血清剥夺的 $\mathrm{PC} 12$ 神经细胞中, 自噬抑 制剂3-甲基嘌呤(3-methyladenine，3-MA)显著降低细 胞凋亡率 ${ }^{[9]}$. 不仅如此, 在大脑中动脉栓塞(middle cerebral artery occlusion, MCAO)的大鼠模型中, 脑室注射 3-MA或溶酶体抑制剂巴非霉素A1后均显著降低了脑 梗死体积 ${ }^{[11,12]}$. 脑室注射自噬诱导剂雷帕霉素(Rapamycin)则加重了大鼠脑缺血再灌注后海马CA1区的神 经损伤 ${ }^{[13]}$. 也有研究报道了在新生小鼠缺氧缺血性神 经损伤中，选择性敲除神经元自噬相关基因 $\operatorname{Atg} 7$ ，抑 制自噬可减少多个脑区的细胞死亡 ${ }^{[14]}$. 虽然上述证据 提示自噬加重了脑缺血损伤，但所用的实验模型存在 较大差异, 自噬是否在复杂的脑缺血各个病理阶段均 发挥神经损伤作用仍有待明确.

与上述观点不同, 近年来越来越多的证据表明, 自 噬在脑缺血过程中发挥了抑制神经元死亡的作用. 有 研究指出, 在小鼠MCAO模型中, 自噬抑制剂3-MA以 及渥曼青霉素(Wortmannin)通过抑制自噬导致神经元 坏死加重, 提示自噬保护了缺血神经元 ${ }^{[15,47,48]}$. 另有研 究表明, 在一过性及永久性MCAO模型中使用雷帕霉 素诱导自噬，均缩小了梗死体积，改善了神经系统预 后, 从而发挥神经保护作用 ${ }^{[17,18]}$. 本课题组研究发现, 自噬在缺血的不同病理阶段中发挥了截然相反的作 用, 在缺血后的复灌过程中, 通过药理学或基因干预手 段抑制自噬均加重神经元损伤 ${ }^{[16]}$, 提示自噬的神经保 护作用存在病理阶段特异性. 不仅如此, 近年来相关 研究也表明, 在脑缺血恢复期, 可观察到自噬泡和自 噬底物的累积，提示自噬流受阻，进而导致缺血损伤 加剧. 而通过3-MA抑制自噬也导致了脑缺血损伤加 重 ${ }^{[19]}$. 因此，自噬对缺血神经元的作用可能与血流中 断的速率、程度、持续时间以及是否发生血流复灌有 关. 近年来多数研究发现, 自噬在缺血神经元中发挥保 护作用, 提示其可能成为潜在的药物研发靶点. 神经元 自噬的发生、调节和作用可能具有精细的时空调节特 点, 也依赖于病理生理微环境的变化.

综上所述, 病理阶段特异性, 缺血时长, 是否存在 复灌等多种因素可能影响自噬在脑缺血中的作用. 神 经元中自噬的调控机制及其在卒中脑内的作用尚需深
入研究.

\section{2 脑内其他细胞中的自噬}

除神经元中的自噬外, 脑内其他细胞的自噬, 例如 星形胶质细胞，小胶质细胞以及血管内皮细胞等也在 缺血脑内发挥重要作用(图2), 以下分别阐述.

(1) 星形胶质细胞. 星形胶质细胞在控制神经环 路、离子稳态、炎症以及神经营养因子稳态等过程中 发挥重要作用. 在体外低氧培养的原代星形胶质细胞 以及动物局灶性脑缺血模型中, 自噬均被激活 ${ }^{[49]}$. 敲 降原代培养的星形胶质细胞Atg5(autophagy related 5) 基因可逆转氧糖剥夺(oxygen and glucose deprivation, OGD)引起的LC3- II 表达水平增加 ${ }^{[50]}$, 敲降星形胶质 细胞的Beclin 1 基因也可以逆转 OGD后miR-30d抑制导 致的自噬上调 ${ }^{[51]}$. 在缺血脑组织的星形胶质细胞中, 研究者也观察到自噬泡的积累 ${ }^{[52]}$. 给予自噬抑制剂 3-MA及巴菲霉素A1可显著减轻缺血损伤下星形胶质 细胞的死亡 ${ }^{[49]}$; 在细胞氧糖剥夺模型中 $3-\mathrm{MA}$ 处理的星 形胶质细胞的自噬显著下调, 调亡以及坏死水平均显 著降低 ${ }^{[53]}$. 以上结果提示在缺血过程中, 星形胶质细 胞的自噬被激活, 并加重损伤. 然而星形胶质细胞中 的自噬激活的时相与程度是否与神经元一致, 其自噬 激活的细胞生物学机制等关键问题均有待回答.

(2) 小胶质细胞. 小胶质细胞是中枢神经系统内 固有的免疫效应细胞, 在中枢神经系统的生理过程中 发挥极其重要的作用. 在脑缺血过程中, 小胶质细胞 可吞噬死亡神经元，从而促进缺血损伤的修复 ${ }^{[5]}$. 在 小鼠脑缺血模型和OGD处理的原代培养小胶质细胞 中均可观察到自噬激活 ${ }^{[55 ~ 57]}$. 进一步研究表明, 缺氧 再灌注可通过ROS调控的Akt/mTOR信号通路激活小 胶质细胞自噬进而诱导小胶质细胞凋亡 ${ }^{[58]}$. 给予 $3-\mathrm{MA}$ 后小胶质细胞自噬水平降低, 并伴有脑梗死体积降低 以及神经症状评分的改善 ${ }^{[55,56]}$, 提示小胶质细胞自噬 可能发挥了加重脑缺血损伤的作用.

然而, 近年来的研究表明, 自噬也可能通过调节小 胶质细胞极化及相关炎症反应发挥抗脑缺血损伤的作 用. 在脑缺血过程中, 小胶质细胞激活并分化为促炎症 的M1型细胞和抗炎症的 $\mathrm{M} 2$ 型. 其中, M1型细胞促进 脑缺血后的二次损伤, 而 $\mathrm{M} 2$ 型细胞则有利于脑缺血后 的康复 ${ }^{[59]}$. 通过 $\mathrm{NH}_{4} \mathrm{Cl}$ 抑制自噬可诱导 $\mathrm{OGD} / \mathrm{R}$ 处理的 $\mathrm{BV}-2$ 小胶质细胞向M1型极化 ${ }^{[60]}$, 从而加重细胞损伤. 


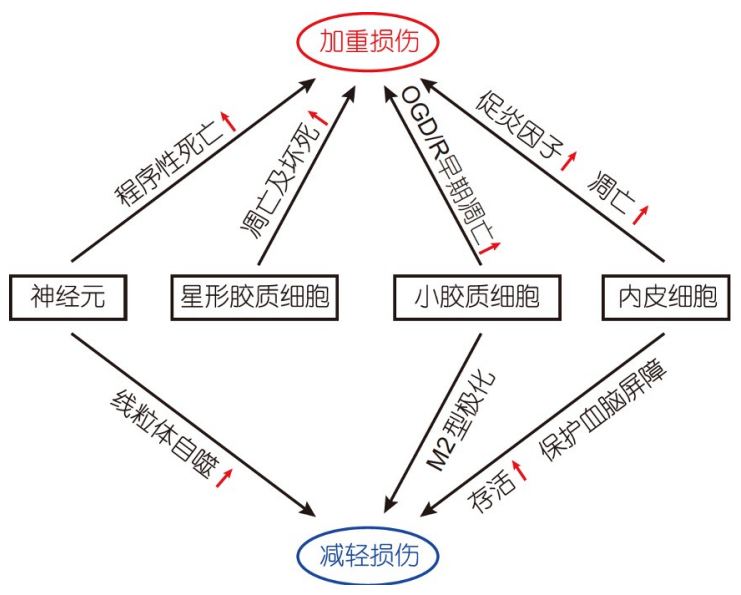

图 2 缺血脑内各类型细胞中自噬的作用. 自噬在脑缺血中 发挥的作用尚存争议: 在脑缺血过程中, 自噬激活可通过促 进神经元程序性死亡、星形胶质细胞调亡或坏死、小胶质 细胞调亡、内皮细胞炎症因子分泌及调亡加重缺血损伤; 也 有证据表明, 自噬激活也可通过激活线粒体自噬发挥神经保 护作用，同时促进小胶质细胞向M2型极化，促进内皮细胞存 活并保护血脑屏障从而减轻缺血损伤(网络版彩图)

Figure 2 Role of autophagy in ischemic stroke. The role of autophagy in ischemic brains is controversial. Autophagy activation may promote neuronal death, apoptosis/necrosis of astrocytes, apoptosis of microglia, secretion of proinflammatory factors, and apoptosis of endothelial cells. On the contrary, evidence supports a neuroprotective role of autophagy, which may reduce ischemic injury by promoting neuronal mitophagy, microglial M2 polarization, endothelial cell survival, and protection of the blood-brain barrier (color online)

通过抑制mTORC1阻断mTOR信号通路、促进自噬, 则可显著减少促炎细胞因子和趋化因子的生成，并阻 止小胶质细胞向 $\mathrm{M} 1$ 型极化, 进而减轻大鼠 $\mathrm{MCAO}$ 处理 后的行为障碍及卒中后炎症反应 ${ }^{[61]}$. 尽管如此，也有 研究表明，通过3-MA抑制自噬可促进小胶质细胞在 OGD处理时向M2型分化，并显著减少炎症反应的发 生 ${ }^{[62]}$. 在永久性大脑中动脉栓塞(permanent middle cerebral artery occlusion, pMCAO)小鼠模型中, 3-MA抑制 小胶质细胞自噬的同时降低了炎症反应，并显著减少 了永久性脑缺血后的梗死体积和神经功能损伤 ${ }^{[56]}$. 有 研究指出, 自噬对小胶质细胞极化的调节受OGD/R处 理时程的影响，尽管在OGD/R早期(复灌 12,24或 48 h) $\mathrm{BV}-2$ 小胶质细胞自噬激活，但在 $\mathrm{OGD} / \mathrm{R}$ 复灌晚期(复 灌72 h) 自噬被抑制，并诱导小胶质细胞向M1型极 化 ${ }^{[60]}$. 然而, 此结论还需在动物模型上进一步验证. 因 此, 自噬对脑缺血过程中小胶质细胞激活及相关炎症 反应的影响仍有待进一步研究.

(3) 脑微血管内皮细胞. 缺血性脑卒中引起血脑屏
障破坏以及血管通透性增加, 继而引发脑水肿. 脑微血 管内皮细胞(brain microvascular endothelial cell, BMVEC) 是脑内特化的内皮细胞, 它们对于维持血管 屏障结构和功能的完整性十分重要. 研究表明, 使用 3-MA抑制自噬可导致缺血后脑微血管内皮细胞调亡 水平增加, 提示在缺血性脑卒中期间, 脑微血管内皮细 胞自噬激活对血脑屏障的完整性有益 ${ }^{[63]}$. 另一研究也 表明, 经由氯喹抑制脑微血管内皮细胞自噬，血脑屏 障通透性以及脑水肿程度都有显著上升 ${ }^{[64]}$. 上述结果 提示, 脑缺血过程中, BMVECs自噬激活可能发挥缺 血保护作用. 然而, 也有研究发现, 3-MA可逆转OGD/ $\mathrm{R}$ 引起的脑血管内皮细胞形态变化, 提高细胞存活率 并抑制调亡 ${ }^{[65]}$. 脑血管内皮细胞敲除A tg 7 抑制自噬也 可显著减少OGD/R弓起的促炎细胞因子表达，从而发 挥神经保护作用 ${ }^{[66]}$. 因此, 脑微血管内皮细胞自噬在 脑缺血中发挥的作用仍有待阐明.

\section{3 缺血脑内的选择性自噬及其作用}

近年来的研究表明, 自噬对底物的存在选择. 通过 降解不同的细胞器和蛋白质, 自噬实现特异性的调节 作用. 在缺血性脑卒中的发生和发展过程中, 自噬可 能通过多种选择性自噬干预细胞内生物学过程, 分别 介绍如下.

\section{1 自噬与线粒体自噬}

线粒体作为细胞的能量中心, 在多种细胞内过程 中发挥重要作用 ${ }^{[67]}$. 在缺血性脑卒中过程中, 细胞受 氧糖剥夺刺激，线粒体发生损伤，从而诱发线粒体自 噬. 线粒体自噬是指通过自噬溶酶体途径清除受损以 及多余线粒体的过程 ${ }^{[28]}$. 自 Lemasters ${ }^{[68]}$ 于 2005 年首次 命名线粒体自噬以来, 越来越多的研究者发现其与多 种疾病密切相关 ${ }^{[69]}$, 干预线粒体自噬是一种潜在的缺 血性脑卒中的治疗策略 ${ }^{[70]}$.

在脑缺血过程中线粒体自噬受多种相关蛋白的调 节，包括第 10 号染色体缺失的磷酸酶和张力蛋白同源 物诱导的假定激酶1(PTEN induced putative kinase 1, PINK1), Parkin, BNIP3L, 含有FUN14结构域包含蛋白 1(FUN14 domain containing 1, FUNDC1)等 ${ }^{[71]}$.

PINK1/Parkin通路调控的线粒体自噬受到广泛关 注. PINK1在线粒体受到去极化刺激后, 触发线粒体自 
噬的起始信号，并将Parkin招募至线粒体，而后Parkin 通过对线粒体蛋白进一步泛素化介导自噬信号的扩 大, 以维持线粒体稳态 ${ }^{[72]}$. 有证据表明, PINK1和Parkin 参与了脑缺血再灌注损伤的病理过程, 氧糖剥夺/复灌 可激活PINK1/Parkin介导的线粒体自噬，从而介导损 伤线粒体的清除 ${ }^{[73]}$. 而PINK1/Parkin通路介导的线粒 体自噬可能受多种条件影响，如缺血复灌，缺氧后处 理可通过激活PINK1/Parkin通路诱导线粒体发生自 噬 ${ }^{[74,75]}$. 本课题组 ${ }^{[29]}$ 研究发现, 通过酸后处理诱导Parkin依赖的自噬可以抵抗脑缺血损伤，并延长再灌注时 间窗。上述研究结果表明, 在脑缺血过程中, 激活 PINK1/Parkin通路介导的线粒体自噬将有助于清除受 损线粒体, 减轻损伤.

FUNDC1在缺氧诱导的线粒体自噬中也发挥重要 作用 ${ }^{[76]}$. FUNDC1可与LC3或自噬相关蛋白 8(autophagy-associated protein, ATG8)结合, 诱导线粒体自 噬 ${ }^{[77]}$. 此外, FUNDC1还通过与视神经萎缩蛋白 (optic atrophy1, OPA1)或(和)线粒体动力相关蛋白 1 (dynamic 1 like, DRP1)的相互作用参与线粒体融合分裂的调 控 ${ }^{[78]}$. 近期研究发现, 组织型纤溶酶原激活剂(tissuetype plasminogenactivator, tPA)通过激活AMPK磷酸化 提高Fundc 1 的表达水平, 改善线粒体功能, 抑制细胞凋 亡, 从而发挥神经保护作用 ${ }^{[79]}$. 关于FUNDC1在脑缺血 中的研究尚少, 目前结果提示FUNDC1介导的线粒体 自噬可能通过清除损伤线粒体 ${ }^{[79]}$, 从而减轻缺血损伤.

BNIP3 和BNIP3L均是仅含 BH3结构域的Bcl-2家 族蛋白, 但与其他诱导细胞调亡蛋白不同, 它们还参与 诱导线粒体自噬 ${ }^{[00,81]}$. 在缺氧的条件下经 $p 53$ 基因的调 控, BNIP3的表达显著增加, 其针定在线粒体外膜, 通 过与LC3互作结构域招募胞浆中的LC3至线粒体表面, 促进自噬小体的形成, 进而诱导线粒体自噬的发生 ${ }^{[82]}$. BNIP3L作为线粒体自噬受体通过与LC3 的结合, 介导 线粒体自噬，参与网织红细胞发育过程中的线粒体清 除 ${ }^{[83]}$, 并在多种疾病中发挥了重要作用. 本课题组 ${ }^{[84]}$ 研究发现, Bnip $3 l$ 基因敲除小鼠展示出更严重的缺血 性脑损伤，而利用慢病毒感染的方法恢复BNIP3L的表 达, 可显著降低脑梗死并改善神经症状评分, 以上结果 提示, BNIP3L介导的线粒体自噬在减轻缺血损伤的过 程中发挥重要作用。此外，在永久性脑缺血过程中, BNIP3L介导的线粒体自噬依赖于其蛋白二聚体形成, BNIP3L二聚体通过蛋白酶体途径降解进而导致线粒
体自噬功能障碍. 利用蛋白酶体抑制剂卡非佐米有效 抑制BNIP3L的降解，从而逆转线粒体自噬障碍并发挥 保护作用 ${ }^{[84]}$. 综上所述, BNIP3L可能成为脑缺血保护 的潜在药物干预靶点, 未来仍需寻找更加精准的 BNIP3L干预方法.

\section{2 内质网自噬}

内质网在新陈代谢和细胞组织中起着至关重要的 作用，其发挥了钻的存储、脂质的生物合成、分泌蛋 白和膜蛋白的成熟和运输等多种作用 ${ }^{[85]}$. 内质网含量 的降解在细胞内稳态中发挥重要作用。内质网自噬 (autophagy of the ER, ER-phagy)是指当内质网功能发 生紊乱时, 细胞激活自噬并选择性清除受损内质网的 过程 ${ }^{[86,87]}$. 在正常生理条件下, 内质网自噬维持在较 低水平, 以维持内质网的稳态. 当发生有害信号刺激 时, 如营养缺乏、未折叠蛋白聚集或致病菌感染, 内 质网自噬即被激活 ${ }^{[88]}$. 内质网应激与多种疾病高度相 关，包括炎症性疾病、自身免疫性疾病以及脑缺血损 伤 ${ }^{[89]}$. 在缺血性卒中发生后, 缺氧打破了钙离子稳态, 导致内质网中的钻离子积累, 进而诱导内质网自噬加 剧, 最终导致细胞死亡 ${ }^{[90]}$. 在大鼠脑缺血模型中, 自噬 抑制剂3-MA加重了脑缺血诱导的内质网应激, 增加了 体外和体内模型中促调亡的caspase-12和procaspase-3 的水平，提示内质网自噬可能导致了缺血损伤的加 重 ${ }^{[91]}$. 此外, 最近有研究表明, 在小鼠的MCAO模型前 给予褪黑素治疗, 可以通过PERK和IREI信号通路抑制 内质网应激依赖的自噬, 从而减轻缺血性卒中后的急 性神经元损伤 ${ }^{[7]}$. 然而, 也有其他研究报告称, 在大鼠 缺血模型中, 抑制内质网自噬也未对缺血损伤产生保 护作用 ${ }^{[92]}$. 在缺血性脑卒中过程中, 内质网自噬的作 用仍需进一步阐明.

\section{4 缺血性脑卒中过程中自噬的调节剂}

多种潜在的缺血性脑卒中治疗药物都以自噬通路 为靶点. 这些药物调控了自噬的不同过程(表 1$)$, 从而 发挥神经保护作用. 第一类药物是靶向自噬的起始阶 段. 雷帕霉素是一种自噬激活剂, 通过抑制mTORC1 继而激活自噬启动的关键成分ULKI复合物来促进自 噬的起始 ${ }^{[93]}$. 有研究报道称, 在小鼠局灶性缺血模型 中使用雷帕霉素可以显著减少脑梗死体积, 改善行为 
李玥等: 自噬在缺血性脑卒中过程中的作用及其调节机制研究进展

表 1 缺血性脑卒中过程中具有潜在神经保护作用的自䓡调节剂

Table 1 Autophagy modulators with potential neuroprotective effects in ischemic stroke

\begin{tabular}{|c|c|c|c|}
\hline 靶向自唑阶段 & 自噬调节剂 & 药理作用 & 参考文献 \\
\hline \multirow{6}{*}{ 起始阶段 } & 雷帕霉素(Rapamycin) & 通过抑制mTORC1激活ULK1复合物, 促进自噬起始 & [93] \\
\hline & 错构瘤蛋白(Hamartin) & 通过抑制mTORC1激活ULK1复合物, 促进自噬起始 & [95] \\
\hline & 二甲双胍(Metformin) & 通过激活AMPK进而激活ULK1复合物, 促进自噬起始 & [96] \\
\hline & 肌肽(Carnosine) & 抑制mTOR磷酸化 & [97] \\
\hline & 芬戈莫德(Fingolimod) & 激活 mTOR/p70S6K 通路, 抑制自跷 & [98] \\
\hline & N-乙酰血清素(N-acetyl-serotonin) & 减少自潄激活 & [99] \\
\hline 自噬泡成核阶段 & 褪黑素(Melatonin) & 抑制Beclin1表达 & {$[100]$} \\
\hline \multirow{2}{*}{ 自噬泡延伸阶段 } & 3-甲基嘌呤(3-MA) & 抑制缺血诱导的LC3表达, 抑制自噬 & {$[12]$} \\
\hline & 贝沙罗汀(Bexarotene) & 抑制自梀泡降解, 促进自噬 & {$[101]$} \\
\hline
\end{tabular}

症状评分 ${ }^{[94]}$. 随后研究发现, TSC1 基因编码错构瘤蛋 白(hamartin)机制与雷帕霉素类似，均作用于自噬的起 始部分发挥神经保护作用 ${ }^{[95]}$. 此外, II 型糖尿病的一 线药物，二甲双胍同样被发现具有抗脑缺血损伤的作 用，其机制为激活AMPK，继而激活ULK1复合物从而 促进自噬的起始 ${ }^{[96]}$. 其他靶向自噬起始的药物包括抑 制mTOR磷酸化的肌肽以及激活mTOR/p70S6K通路 从而抑制自噬的芬戈莫德(Fingolimod) 等 ${ }^{[97,98]}$. 另有研 究者认为, $\mathrm{N}$-乙酰血清素通过激活自噬发挥了抗脑缺 血损伤的作用 ${ }^{[99]}$. 第二类药物靶向自噬泡的成核或延 伸阶段, 包括褪黑素、3-MA以及贝沙罗汀. 裉黑素抗 脑缺血的机制与其前体物质N-乙酰血清素有所不同, 它可通过抑制Beclin1表达影响自噬泡成核，从而减轻 脑缺血导致的神经元损伤 ${ }^{[7,100]}$. 3-MA是一种经典的自 噬抑制剂, 通过抑制缺血诱导的LC3表达，而抑制自噬 泡的延伸，从而减少缺血性脑卒中中自噬诱导的损 伤 ${ }^{[12]}$. 贝沙罗汀用于治疗皮肤癌，其通过促进自噬泡 的延伸过程，促进自噬，继而发挥神经保护作用 ${ }^{[01]}$. 此外, 维甲酸、伏立诺他具有激活自噬的作用, 但上 述药物在脑缺血领域未有研究 ${ }^{[102]}$, 这些药物是否能用 于缺血性脑卒中的治疗值得进一步深入探讨.

\section{5 展望}

自噬在缺血性脑卒中的发生与发展过程中发挥重 要作用, 但缺血脑内多种细胞中自噬, 尤其是选择性自 噬的发生及调节机制尚未被完全阐明，自噬在脑缺血 过程中发挥的作用仍存在争议. 在研究过程中, 实验条 件的差异, 包括动物种类/年龄、细胞/动物模型、缺血 时间以及强度等, 可能导致不同的结论. 自噬调节的工 具药物特异性不强, 特别是被当做自噬抑制剂使用的 3-MA在特定条件下可能具有自噬促进作用 ${ }^{[103]}$, 为实 验结果的解释带来了困难. 不仅如此, 多种选择性自噬 在缺血脑内可能发挥不同作用, 但多数选择性自噬的 分子机制及相互作用尚未被完全阐明, 因此也缺乏特 异研究某种选择性自噬的有力工具. 上述问题还需要 不断深入研究. 自噬调节剂可能是潜在的缺血性脑卒 中干预药物, 但通过调节自噬进而干预缺血性脑卒中 可能还存在诸多困难. 由于脑内自噬的作用机制尚不 完全清楚, 目前仍缺乏明确的自噬干预靶点. 特别是自 噬具有重要生理作用, 这些药物潜在的不良反应仍需 得到重视. 另一方面, 一些已在临床使用且较为安全的 药物近来被发现具有自噬调节作用, 这些药物是否能 用于缺血性脑卒中的治疗值得进一步探索.

\section{参考文献}

1 Gou X, Xu D, Li F, et al. Pyroptosis in stroke-new insights into disease mechanisms and therapeutic strategies. J Physiol Biochem, 2021, doi: 10.1007/s13105-021-00817-w

2 Tuo Q Z, Zhang S T, Lei P. Mechanisms of neuronal cell death in ischemic stroke and their therapeutic implications. Med Res Rev, 2021, doi: 
10.1002/med.21817

3 Galkin A. Brain ischemia/reperfusion injury and mitochondrial complex I damage. Biochem Moscow, 2019, 84: 1411-1423

4 Dikic I, Elazar Z. Mechanism and medical implications of mammalian autophagy. Nat Rev Mol Cell Biol, 2018, 19: 349-364

5 Wang P, Shao B Z, Deng Z, et al. Autophagy in ischemic stroke. Prog Neurobiol, 2018, 163-164: 98-117

6 Wang M, Liang X, Cheng M, et al. Homocysteine enhances neural stem cell autophagy in in vivo and in vitro model of ischemic stroke. Cell Death Dis, 2019, 10: 561

7 Feng D, Wang B, Wang L, et al. Pre-ischemia melatonin treatment alleviated acute neuronal injury after ischemic stroke by inhibiting endoplasmic reticulum stress-dependent autophagy via PERK and IRE1 signalings. J Pineal Res, 2017, 62: e12395

8 Wang M M, Zhang M, Feng Y S, et al. Electroacupuncture inhibits neuronal autophagy and apoptosis via the PI3K/AKT pathway following ischemic stroke. Front Cell Neurosci, 2020, 14: 134

9 Uchiyama Y. Autophagic cell death and its execution by lysosomal cathepsins. Arch Histol Cytol, 2001, 64: 233-246

10 Shi R, Weng J, Zhao L, et al. Excessive autophagy contributes to neuron death in cerebral ischemia. CNS Neurosci Ther, 2012, 18: 250-260

11 Puyal J, Vaslin A, Mottier V, et al. Postischemic treatment of neonatal cerebral ischemia should target autophagy. Ann Neurol, 2009, 66: 378389

12 Wen Y D, Sheng R, Zhang L S, et al. Neuronal injury in rat model of permanent focal cerebral ischemia is associated with activation of autophagic and lysosomal pathways. Autophagy, 2008, 4: 762-769

13 Dong F, Yao R, Yu H, et al. Neuroprotection of Ro25-6981 against ischemia/reperfusion-induced brain injury via inhibition of autophagy. Cell Mol Neurobiol, 2017, 37: 743-752

14 Xie C, Ginet V, Sun Y, et al. Neuroprotection by selective neuronal deletion of Atg7 in neonatal brain injury. Autophagy, 2016, 12: 410-423

15 Carloni S, Buonocore G, Balduini W. Protective role of autophagy in neonatal hypoxia-ischemia induced brain injury. Neurobiol Dis, 2008, 32 : 329-339

16 Zhang X, Yan H, Yuan Y, et al. Cerebral ischemia-reperfusion-induced autophagy protects against neuronal injury by mitochondrial clearance. Autophagy, 2013, 9: 1321-1333

17 Buckley K M, Hess D L, Sazonova I Y, et al. Rapamycin up-regulation of autophagy reduces infarct size and improves outcomes in both permanent MCAL, and embolic MCAO, murine models of stroke. Exp Trans Stroke Med, 2014, 6: 8

18 Li Q, Zhang T, Wang J, et al. Rapamycin attenuates mitochondrial dysfunction via activation of mitophagy in experimental ischemic stroke. Biochem Biophys Res Commun, 2014, 444: 182-188

19 Chen X, Lin S, Gu L, et al. Inhibition of miR-497 improves functional outcome after ischemic stroke by enhancing neuronal autophagy in young and aged rats. Neurochem Int, 2019, 127: 64-72

20 Liu Y, Xue X, Zhang H, et al. Neuronal-targeted TFEB rescues dysfunction of the autophagy-lysosomal pathway and alleviates ischemic injury in permanent cerebral ischemia. Autophagy, 2019, 15: 493-509

21 Wang X S, Yue J, Hu L N, et al. Activation of G protein-coupled receptor 30 protects neurons by regulating autophagy in astrocytes. Glia, 2020, 68: $27-43$

22 Zhi S M, Fang G X, Xie X M, et al. Melatonin reduces OGD/R-induced neuron injury by regulating redox/inflammation/apoptosis signaling. Eur Rev Med Pharmacol Sci, 2020, 24: 1524-1536

23 Pan Q, Liu Y, Wang G, et al. MTMR14 protects against cerebral stroke through suppressing PTEN-regulated autophagy. Biochem Biophys Res Commun, 2020, 529: 1045-1052

24 Cai W, Zhang K, Li P, et al. Dysfunction of the neurovascular unit in ischemic stroke and neurodegenerative diseases: an aging effect. Ageing Res Rev, 2017, 34: 77-87

25 Radak D, Katsiki N, Resanovic I, et al. Apoptosis and acute brain ischemia in ischemic stroke. Curr Vasc Pharmacol, 2017, 15: 115-122

26 Stanzione R, Cotugno M, Bianchi F, et al. Pathogenesis of ischemic stroke: role of epigenetic mechanisms. Genes, 2020, 11: 89

27 Wu Q J, Tymianski M. Targeting NMDA receptors in stroke: new hope in neuroprotection. Mol Brain, 2018, 11: 15

28 Anzell A R, Maizy R, Przyklenk K, et al. Mitochondrial quality control and disease: insights into ischemia-reperfusion injury. Mol Neurobiol, 2018, 55: 2547-2564

29 Shen Z, Zheng Y, Wu J, et al. PARK2-dependent mitophagy induced by acidic postconditioning protects against focal cerebral ischemia and extends the reperfusion window. Autophagy, 2017, 13: 473-485 
30 Chouchani E T, Pell V R, Gaude E, et al. Ischaemic accumulation of succinate controls reperfusion injury through mitochondrial ROS. Nature, 2014, 515: 431-435

31 Filomeni G, De Zio D, Cecconi F. Oxidative stress and autophagy: the clash between damage and metabolic needs. Cell Death Differ, 2015, 22: $377-388$

32 Yu J, Wang W N, Matei N, et al. Ezetimibe attenuates oxidative stress and neuroinflammation via the AMPK/Nrf2/TXNIP pathway after MCAO in rats. Oxid Med Cell Longev, 2020, 2020: 1-14

33 Jiang T, Harder B, Rojo de la Vega M, et al. p62 links autophagy and Nrf2 signaling. Free Radic Biol Med, 2015, 88: 199-204

34 Li L, Tan J, Miao Y, et al. ROS and autophagy: interactions and molecular regulatory mechanisms. Cell Mol Neurobiol, 2015, 35: 615-621

35 Bellot G, Garcia-Medina R, Gounon P, et al. Hypoxia-induced autophagy is mediated through hypoxia-inducible factor induction of BNIP3 and BNIP3L via their BH3 domains. Mol Cell Biol, 2009, 29: 2570-2581

36 Semenza G L. Hypoxia-inducible factor 1: regulator of mitochondrial metabolism and mediator of ischemic preconditioning. Biochim Biophys Acta, 2011, 1813: 1263-1268

37 Zhang H, Bosch-Marce M, Shimoda L A, et al. Mitochondrial autophagy is an HIF-1-dependent adaptive metabolic response to hypoxia. J Biol Chem, 2008, 283: 10892-10903

38 Cheung E C, Ludwig R L, Vousden K H. Mitochondrial localization of TIGAR under hypoxia stimulates HK2 and lowers ROS and cell death. Proc Natl Acad Sci USA, 2012, 109: 20491-20496

39 Bensaad K, Cheung E C, Vousden K H. Modulation of intracellular ROS levels by TIGAR controls autophagy. EMBO J, 2009, 28: 3015-3026

40 Crighton D, Wilkinson S, O'Prey J, et al. DRAM, a p53-induced modulator of autophagy, is critical for apoptosis. Cell, 2006, 126: 121-134

41 Chamorro Á, Dirnagl U, Urra X, et al. Neuroprotection in acute stroke: targeting excitotoxicity, oxidative and nitrosative stress, and inflammation. Lancet Neurol, 2016, 15: 869-881

42 Maiti P, Peruzzaro S, Kolli N, et al. Transplantation of mesenchymal stem cells overexpressing interleukin-10 induces autophagy response and promotes neuroprotection in a rat model of TBI. J Cell Mol Med, 2019, 23: 5211-5224

43 Netea-Maier R T, Plantinga T S, van de Veerdonk F L, et al. Modulation of inflammation by autophagy: consequences for human disease. Autophagy, 2016, 12: 245-260

44 Fu C, Zhang X, Lu Y, et al. Geniposide inhibits NLRP3 inflammasome activation via autophagy in BV-2 microglial cells exposed to oxygenglucose deprivation/reoxygenation. Int Immunopharmacol, 2020, 84: 106547

45 Nitatori T, Sato N, Waguri S, et al. Delayed neuronal death in the CA1 pyramidal cell layer of the gerbil hippocampus following transient ischemia is apoptosis. J Neurosci, 1995, 15: 1001-1011

46 Tian F F, Deguchi K, Yamashita T, et al. In vivo imaging of autophagy in a mouse stroke model. Autophagy, 2010, 6: 1107-1114

47 Wang P, Guan Y F, Du H, et al. Induction of autophagy contributes to the neuroprotection of nicotinamide phosphoribosyltransferase in cerebral ischemia. Autophagy, 2012, 8: 77-87

48 Wang P, Xu T Y, Wei K, et al. ARRB1//-arrestin-1 mediates neuroprotection through coordination of BECN1-dependent autophagy in cerebral ischemia. Autophagy, 2014, 10: 1535-1548

49 Qin A P, Liu C F, Qin Y Y, et al. Autophagy was activated in injured astrocytes and mildly decreased cell survival following glucose and oxygen deprivation and focal cerebral ischemia. Autophagy, 2010, 6: 738-753

50 Zhou X Y, Luo Y, Zhu Y M, et al. Inhibition of autophagy blocks cathepsins-tBid-mitochondrial apoptotic signaling pathway via stabilization of lysosomal membrane in ischemic astrocytes. Cell Death Dis, 2017, 8: e2618

51 Zhao F, Qu Y, Wang H, et al. The effect of miR-30d on apoptosis and autophagy in cultured astrocytes under oxygen-glucose deprivation. Brain Res, 2017, 1671: 67-76

52 Zhang T, Liu X, Li Q, et al. Exacerbation of ischemia-induced amyloid- $\beta$ generation by diabetes is associated with autophagy activation in mice brain. Neurosci Lett, 2010, 479: 215-220

53 Kasprowska D, Machnik G, Kost A, et al. Time-dependent changes in apoptosis upon autophagy inhibition in astrocytes exposed to oxygen and glucose deprivation. Cell Mol Neurobiol, 2017, 37: 223-234

54 Mo Y, Sun Y Y, Liu K Y. Autophagy and inflammation in ischemic stroke. Neural Regen Res, 2020, 15: 1388-1396

55 Yang Z, Zhang N, Shen H, et al. Microglial activation with reduction in autophagy limits white matter lesions and improves cognitive defects during cerebral hypoperfusion. Curr Neurovasc Res, 2014, 11: 223-229 
56 Yang Z, Zhong L, Zhong S, et al. Hypoxia induces microglia autophagy and neural inflammation injury in focal cerebral ischemia model. Exp Mol Pathol, 2015, 98: 219-224

57 Chen Y, Li Z. Protective effects of propofol on rats with cerebral ischemia-reperfusion injury via the PI3K/Akt pathway. J Mol Neurosci, 2021, 71: $810-820$

58 Chen C M, Wu C T, Yang T H, et al. Green tea catechin prevents hypoxia/reperfusion-evoked oxidative stress-regulated autophagy-activated apoptosis and cell death in microglial cells. J Agric Food Chem, 2016, 64: 4078-4085

59 Jiang C T, Wu W F, Deng Y H, et al. Modulators of microglia activation and polarization in ischemic stroke (Review). Mol Med Rep, 2020, 21: 2006-2018

60 Xia C Y, Zhang S, Chu S F, et al. Autophagic flux regulates microglial phenotype according to the time of oxygen-glucose deprivation/ reperfusion. Int Immunopharmacol, 2016, 39: 140-148

61 Li D, Wang C, Yao Y, et al. mTORC1 pathway disruption ameliorates brain inflammation following stroke via a shift in microglia phenotype from M1 type to M2 type. FASEB J, 2016, 30: 3388-3399

62 Jiang M, Wang H, Jin M, et al. Exosomes from miR-30d-5p-ADSCs reverse acute ischemic stroke-induced, autophagy-mediated brain injury by promoting M2 microglial/macrophage polarization. Cell Physiol Biochem, 2018, 47: 864-878

$63 \mathrm{Li} \mathrm{H}$, Gao A, Feng D, et al. Evaluation of the protective potential of brain microvascular endothelial cell autophagy on blood-brain barrier integrity during experimental cerebral ischemia-reperfusion injury. Transl Stroke Res, 2014, 5: 618-626

64 Fang L, Li X, Zhong Y, et al. Autophagy protects human brain microvascular endothelial cells against methylglyoxal-induced injuries, reproducible in a cerebral ischemic model in diabetic rats. J Neurochem, 2015, 135: 431-440

65 Yang G, Wang N, Seto S W, et al. Hydroxysafflor yellow a protects brain microvascular endothelial cells against oxygen glucose deprivation/ reoxygenation injury: involvement of inhibiting autophagy via class I PI3K/Akt/mTOR signaling pathway. Brain Res Bull, 2018, 140: 243-257

66 Wang H J, Wei J Y, Liu D X, et al. Endothelial Atg7 deficiency ameliorates acute cerebral injury induced by ischemia/reperfusion. Front Neurol, 2018, 9: 998

67 Pfanner N, Warscheid B, Wiedemann N. Mitochondrial proteins: from biogenesis to functional networks. Nat Rev Mol Cell Biol, 2019, 20: 267284

68 Lemasters J J. Selective mitochondrial autophagy, or mitophagy, as a targeted defense against oxidative stress, mitochondrial dysfunction, and aging. Rejuvenation Res, 2005, 8: 3-5

69 Guan R, Zou W, Dai X, et al. Mitophagy, a potential therapeutic target for stroke. J Biomed Sci, 2018, 25: 87

70 Yang J L, Mukda S, Chen S D. Diverse roles of mitochondria in ischemic stroke. Redox Biol, 2018, 16: 263-275

71 Novak I. Mitophagy: a complex mechanism of mitochondrial removal. Antioxid Redox Signal, 2012, 17: 794-802

72 Eiyama A, Okamoto K. PINK1/Parkin-mediated mitophagy in mammalian cells. Curr Opin Cell Biol, 2015, 33: 95-101

73 Lan R, Wu J T, Wu T, et al. Mitophagy is activated in brain damage induced by cerebral ischemia and reperfusion via the PINK1/Parkin/p62 signalling pathway. Brain Res Bull, 2018, 142: 63-77

$74 \mathrm{Wu}$ X, Li X, Liu Y, et al. Hydrogen exerts neuroprotective effects on OGD/R damaged neurons in rat hippocampal by protecting mitochondrial function via regulating mitophagy mediated by PINK1/Parkin signaling pathway. Brain Res, 2018, 1698: 89-98

75 Wen H, Li L, Zhan L, et al. Hypoxic postconditioning promotes mitophagy against transient global cerebral ischemia via PINK1/Parkin-induced mitochondrial ubiquitination in adult rats. Cell Death Dis, 2021, 12: 630

76 Lim Y, Rubio-Peña K, Sobraske P J, et al. Fndc-1 contributes to paternal mitochondria elimination in C. elegans. Dev Biol, 2019, 454: 15-20

77 Liu L, Sakakibara K, Chen Q, et al. Receptor-mediated mitophagy in yeast and mammalian systems. Cell Res, 2014, 24: 787-795

78 Chen M, Chen Z, Wang Y, et al. Mitophagy receptor FUNDC1 regulates mitochondrial dynamics and mitophagy. Autophagy, 2016, 12: 689702

79 Cai Y, Yang E, Yao X, et al. FUNDC1-dependent mitophagy induced by tPA protects neurons against cerebral ischemia-reperfusion injury. Redox Biol, 2021, 38: 101792

80 Zhang J, Ney P A. Role of BNIP3 and NIX in cell death, autophagy, and mitophagy. Cell Death Differ, 2009, 16: 939-946

81 Ney P A. Mitochondrial autophagy: origins, significance, and role of BNIP3 and NIX. Biochim Biophys Acta, 2015, 1853: 2775-2783

82 Ishihara M, Urushido M, Hamada K, et al. Sestrin-2 and BNIP3 regulate autophagy and mitophagy in renal tubular cells in acute kidney injury. Am J Physiol Renal Physiol, 2013, 305: F495-F509 
83 McLelland G L, Soubannier V, Chen C X, et al. Parkin and PINK1 function in a vesicular trafficking pathway regulating mitochondrial quality control. EMBO J, 2014, 33: 282-295

84 Wu X, Zheng Y, Liu M, et al. BNIP3L/NIX degradation leads to mitophagy deficiency in ischemic brains. Autophagy, 2021, 17: 1934-1946

85 Schwarz D S, Blower M D. The endoplasmic reticulum: structure, function and response to cellular signaling. Cell Mol Life Sci, 2016, 73: 7994

86 Bernales S, McDonald K L, Walter P. Autophagy counterbalances endoplasmic reticulum expansion during the unfolded protein response. PLoS Biol, 2006, 4: e423

87 Schuck S, Prinz W A, Thorn K S, et al. Membrane expansion alleviates endoplasmic reticulum stress independently of the unfolded protein response. J Cell Biol, 2009, 187: 525-536

88 Grumati P, Dikic I, Stolz A. ER-phagy at a glance. J Cell Sci, 2018, 131

89 Hetz C. The unfolded protein response: controlling cell fate decisions under ER stress and beyond. Nat Rev Mol Cell Biol, 2012, 13: 89-102

90 Nakka V P, Gusain A, Raghubir R. Endoplasmic reticulum stress plays critical role in brain damage after cerebral ischemia/reperfusion in rats. Neurotox Res, 2010, 17: 189-202

91 Sheng R, Liu X Q, Zhang L S, et al. Autophagy regulates endoplasmic reticulum stress in ischemic preconditioning. Autophagy, 2012, 8: 310325

92 Hadley G, Neuhaus A A, Couch Y, et al. The role of the endoplasmic reticulum stress response following cerebral ischemia. Int J Stroke, 2018, 13: $379-390$

93 Hadley G, Beard D J, Couch Y, et al. Rapamycin in ischemic stroke: old drug, new tricks? J Cereb Blood Flow Metab, 2019, 39: 20-35

94 Beard D J, Hadley G, Thurley N, et al. The effect of rapamycin treatment on cerebral ischemia: a systematic review and meta-analysis of animal model studies. Int J Stroke, 2019, 14: 137-145

95 Papadakis M, Hadley G, Xilouri M, et al. Tsc1 (hamartin) confers neuroprotection against ischemia by inducing autophagy. Nat Med, 2013, 19: 351-357

96 Jiang T, Yu J T, Zhu X C, et al. Acute metformin preconditioning confers neuroprotection against focal cerebral ischaemia by pre-activation of AMPK-dependent autophagy. Br J Pharmacol, 2014, 171: 3146-3157

97 Baek S H, Noh A R, Kim K A, et al. Modulation of mitochondrial function and autophagy mediates carnosine neuroprotection against ischemic brain damage. Stroke, 2014, 45: 2438-2443

98 Li X, Wang M H, Qin C, et al. Fingolimod suppresses neuronal autophagy through the mTOR/p70S6K pathway and alleviates ischemic brain damage in mice. PLoS ONE, 2017, 12: e0188748

99 Zhou H, Wang J, Jiang J, et al. N-acetyl-serotonin offers neuroprotection through inhibiting mitochondrial death pathways and autophagic activation in experimental models of ischemic injury. J Neurosci, 2014, 34: 2967-2978

100 Zheng Y, Hou J, Liu J, et al. Inhibition of autophagy contributes to melatonin-mediated neuroprotection against transient focal cerebral ischemia in rats. J Pharmacol Sci, 2014, 124: 354-364

101 Huuskonen M T, Loppi S, Dhungana H, et al. Bexarotene targets autophagy and is protective against thromboembolic stroke in aged mice with tauopathy. Sci Rep, 2016, 6: 33176

102 Galluzzi L, Bravo-San Pedro J M, Levine B, et al. Pharmacological modulation of autophagy: therapeutic potential and persisting obstacles. Nat Rev Drug Discov, 2017, 16: 487-511

103 Wu Y T, Tan H L, Shui G, et al. Dual role of 3-methyladenine in modulation of autophagy via different temporal patterns of inhibition on class I and III phosphoinositide 3-kinase. J Biol Chem, 2010, 285: 10850-10861 


\title{
The role of autophagy and related mechanisms in ischemic brain injury: an update
}

\author{
LI Yue ${ }^{1}$, ZHENG WanQing ${ }^{1}$, PAN Ling ${ }^{1}$, LIU MengRu ${ }^{1}$, ZHOU XinYu ${ }^{1}$, \\ CHEN Zhong ${ }^{1,2} \&$ ZHANG XiangNan ${ }^{1}$
}

\begin{abstract}
1 Institute of Pharmacology and Toxicology, College of Pharmaceutical Sciences, Zhejiang University, Hangzhou 310058, China; 2 Key Laboratory of Neuropharmacology and Translational Medicine of Zhejiang Province, School of Pharmaceutical Sciences, Zhejiang Chinese Medical University, Hangzhou 310053, China
\end{abstract}

Ischemic stroke is a severe cerebrovascular disease that is associated with high morbidity, disability, and mortality. Ischemia activates neuronal autophagy; however, the contribution of autophagy in ischemic brains remains controversial. Autophagy is an intracellular process by which cells degrade damaged organelles and macromolecules via lysosomal pathways. Emerging evidence suggests that autophagy ameliorates ischemic stroke through a variety of mechanisms. Here, we summarize the pathological events that may trigger autophagy in ischemic brains and discuss how autophagy may impact the process of ischemic stroke. We also review the regulatory mechanisms of autophagy in the process of cerebral ischemia and summarize the bioactive compounds that are known to regulate autophagy in ischemic brains. We aim to update the knowledge regarding autophagy in cerebral ischemia and provide new insights into novel drugs for stroke therapy.

ischemic stroke, autophagy, mitophagy

doi: $10.1360 /$ SSV-2021-0194

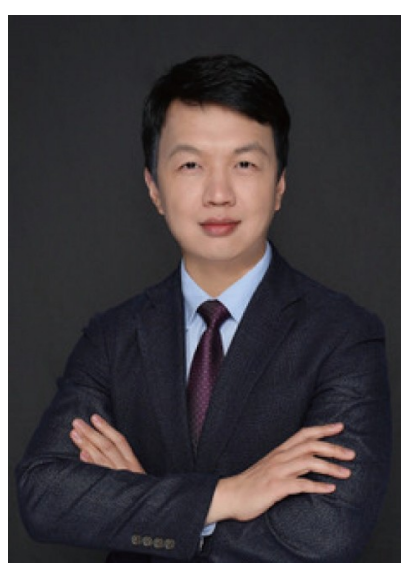

张翔南, 浙江大学药学院教授, 博士生导师. 2010年于浙江大学药理学专业获得博 士学位. 2018年获得国家自然科学基金“优秀青年科学基金”，2019年入选浙江省 “万人计划”青年拔尖人才. 曾获中国药理学会“施维雅青年药理学家”奖、首届全 国临床药学专业青年教师教学基本功竞赛一等奖和浙江省高校第九届青年教师教 学技能竞赛特等奖. 主要从事缺血性脑损伤过程中线粒体自噬的作用及其调控机 制研究, 并在此基础上进行相关药物调控靶点的研究. 相关成果已在高水平学术期 刊上发表论文 20 余篇, 作为参与者获得教育部自然科学奖一等奖. 